\title{
Simulation and Performance Analysis of Interleave Division Multiple Access (IDMA) in Comparision with Code Division Multiple Access (CDMA)
}

\author{
D. Sony ${ }^{1}$, P. Keerthi ${ }^{2 *}$, O. Aditya ${ }^{3} \&$ N. Sravya ${ }^{4}$ \\ ${ }^{I}$ Lecturer, Dept. of ECE, Chaitanya Bharathi Institute of Technology, Osmania University, Hyderabad, Telangana, India. \\ ${ }^{2,3,4}$ UG Scholar, Dept. of ECE, Chaitanya Bharathi Institute of Technology, Osmania University, Hyderabad, Telangana, India.
}

Copyright: (92021 D.Sony et al. This is an open access article distributed under the terms of the Creative Commons Attribution License, which permits unrestricted use, distribution, and reproduction in any medium, provided the original author and source are credited.

\section{ABSTRACT}

This paper provides a study of Interleave division multiple access (IDMA) system by implementing in MATLAB and comparison with Code division multiple access (CDMA) in terms of performance. By using MATLAB we can analyze data, develop algorithms, create models and applications as it is more familiarised to engineers and scientists. CDMA is a technology used in 4G. This system provides multiple access communication capabilities. In CDMA as the number of users increase, the overall quality of performance decreases. So to overcome the drawbacks in CDMA, a new technology IDMA has been developed with the inheritance and advancement of CDMA. IDMA is a technology that is explored world-wide in 5G Communication system. IDMA is a multiuser scheme where users are separated by unique interleaver sequences.

Keywords: Code division multiple access (CDMA), Interleave division multiple access (IDMA), Bit error rate (BER), Signal to noise ratio (SNR).

\section{Introduction}

Wireless Communication is a method of transmitting information from one point to other, without using any connection like wires, cables or any physical medium. Generally, in a communication system, information is transmitted from transmitter to receiver that are placed over a limited distance. In our project we will implement IDMA system and CDMA system using MATLAB and then the performance of these systems are compared in terms of Bit Error Rate (BER) and Signal to Noise Ratio (SNR). The objective of our project is implementation of CDMA System and IDMA System using MATLAB. Then analysis of Bit error Rate Performance of IDMA system over AWGN channel. Then Comparing Bit error Rate Performance of both IDMA and CDMA systems over Additive white gaussian noise (AWGN) channel.

\section{Introduction to IDMA}

In IDMA Interleavers are used to differentiate the signals on entire bandwidth spectrum [1],[2,],[3]. Since different users are using different interleavers it optimizes ISI. Role of the interleaver is to break low weight input sequences, and hence increase the code free Hamming distance or reduce the number of code words with small distances in the code distance spectrum. In interleaving mechanism, At the transmitter end, the input data streams rearranges itself such that consecutive data bits are split among different blocks and is swapped in a known pattern amongst them.

At the receiver end, the interleaved data is arranged back into the original sequence with the help of de-inter leaver. As a result of interleaving, correlated noise introduced in the transmission channel appears to be statistically independent at the receiver and thus allows better error correction. IDMA offers a number of features: Rate/power adaptation, MIMO According to Shannon, typical sequences are generated and superimposed, fast fading, frequency-selective fading, complexity is linear with respect to the number of layers, number of chips/number of users, number of receiver antennas, number of channel taps, and the number of iterations, delivers reliable 


\section{A.}

Soft-output information, Resource allocation, Low delay. A 4G system is expected to provide a comprehensive and secure. all possible solution where facilities such as IP telephony, ultra-broadband internet access, gaming services and streamed multimedia may be provide to users [4],[5].

SNR is calculated as,

$$
S N R=\frac{\text { Signal power }}{\text { Noise power }}
$$

\section{BER is calculated as,}

$$
B E R=\frac{\text { Error in received bits }}{\text { Total no. of bits }}
$$

\section{Random Interleaver}

Random Interleaver illustration is shown in the below figure 1. Random interleavers can interleave the data of different users with different pattern. These patterns of scrambling the data of users are to be generated [6,][7]. These scrambling of data causes burst error of the channel are randomized to the receiver end.With the help of random permutation, the user specific random interleaver is used to rearrange the elements of its input vector.

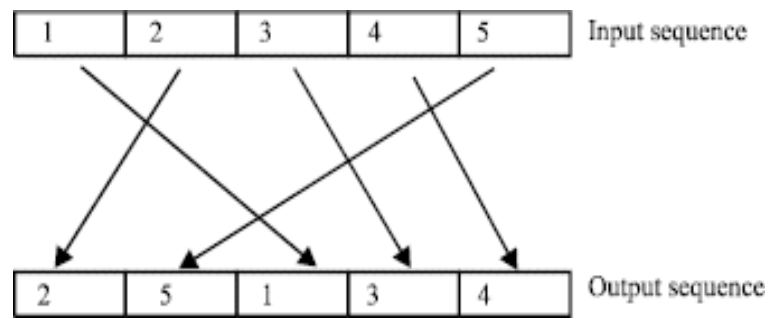

Fig.1. Random Interleaver

These incoming data can rearrange using a series of generated permuter indices. A permuter is generally a device that helps to generate the pseudo-random permutation of given memory addresses. These data is arranged in order of pseudo-random order of memory addresses. When random interleavers are work for the purpose of user separation, then it requires a lot of memory space will be at the transmitter and receiver ends for storage purpose. Hence considerable amount of bandwidth will be consumed for transmission of all these interleaver.

Thus at receiver end, computational complexity will be increases. After randomization of the burst error that has to rearrange the whole block And IDMA provides good security compared to CDMA of the data afterwards it can now be easily detected and corrected. Spreading is one of the essential characteristic of random interleavers [8],[9].

So it is observant that the data of a certain sequence is being randomly distributed which is why it is called RANDOM interleaving. Now as of the interleaving aspects these sequences are distributed. The sequence of the data is a randomly generated code and this random code is a PN sequence i.e. Pseudo random Noise.

The main concern in any communication is that the security we can get over a communication media and the privacy. As it's a PN sequence which is a spontaneous that only the sender knows the sequence as a result the 


\section{AJA5T}

Asian Journal of Applied Science and Technology (AJAST)

Volume 5, Issue 2, Pages 90-94, April-June 2021

receiver must know the source code which is why this was one of the main concern for the researchers supporting in the context of all new adapting techniques and methodologies. However the data encrypted by PN sequence method can be implemented by using MATLAB where initially the data is being spread and multiplied with the sequence.

\section{Methodology}

STEP 1 :- Implementation of CDMA Using matlab and calculating BER and SNR.

STEP 2 :- Implementation of IDMA using Random interleaver in matlab and calculating BER and SNR.

STEP 3 :- Comparison of IDMA and CDMA.

\section{Results}

We have observed BER (Bit Error Rate) of IDMA (Inter Divison Multiple Access) is less compared to CDMA (Code Division Multiple Access). And Signal-to-Noise ratio (SNR) of IDMA (Inter Divison Multiple Access) is better compared to CDMA (Code Division Multiple Access). IDMA eliminates ISI (Inter symbol Interference) and mitigation effect of multi user interface.

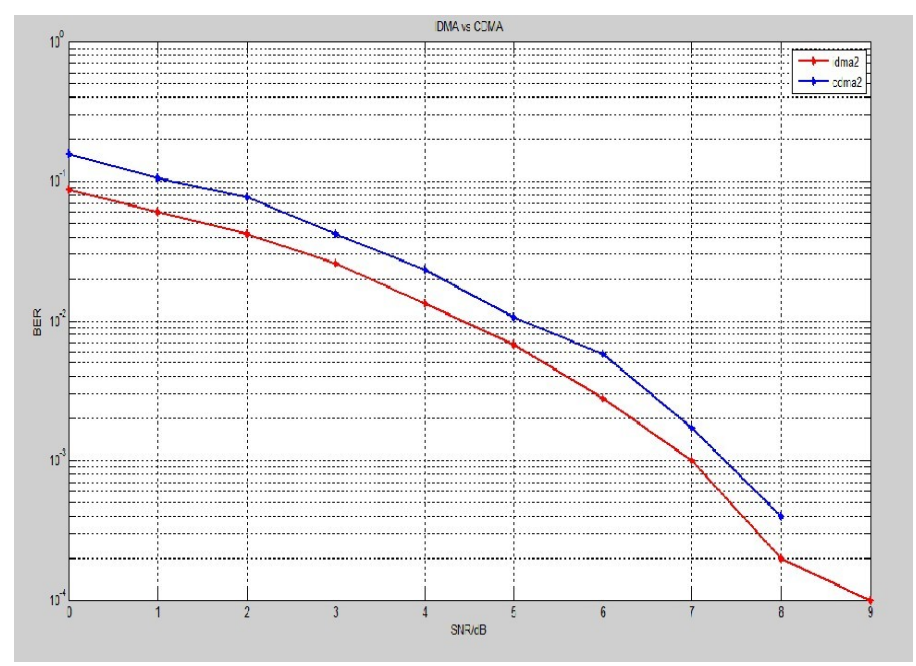

Fig.2. For 2 users

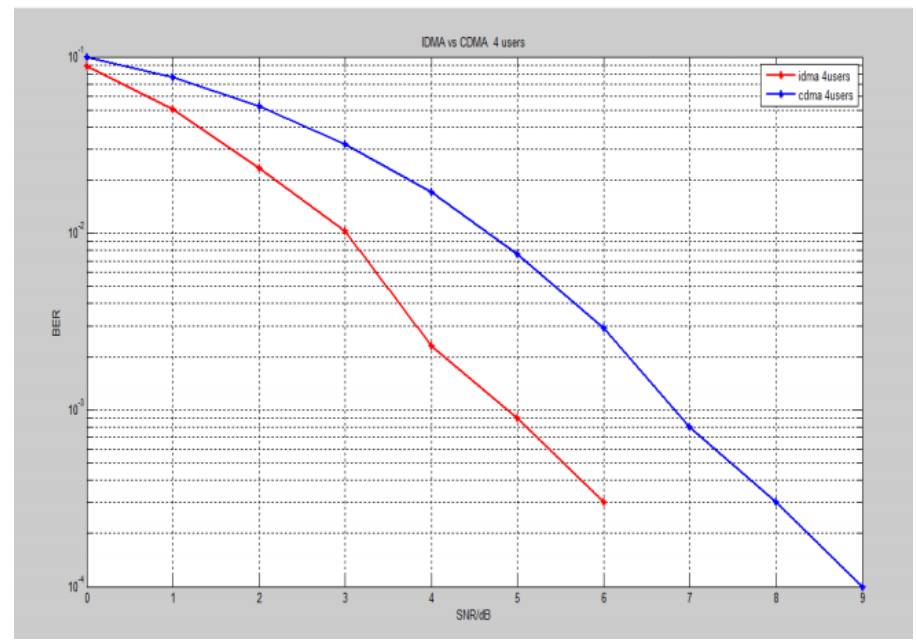

Fig.3. For 4 users 
Table 1. CDMA

\begin{tabular}{|c|c|c|c|c|}
\hline Users & BER & SNR & BER & SNR \\
\hline 2 Users & 0.0105 & 0 & 0.0874 & 0 \\
& 0.0015 & 8 & 0.0001 & 8 \\
\hline \multirow{2}{*}{ 4 Users } & 0.0150 & 0 & 0.0743 & 0 \\
& 0.0012 & 8 & 0.0002 & 8 \\
\hline
\end{tabular}

Table 2. IDMA

\begin{tabular}{|c|c|c|c|c|}
\hline Users & BER & SNR & BER & SNR \\
\hline \multirow{2}{*}{ 2 Users } & 0.095 & 0 & 0.0743 & 0 \\
& 0.0015 & 8 & 0.0002 & 8 \\
\hline \multirow{2}{*}{ 4 Users } & 0.100 & 0 & 0.025 & 0 \\
& 0.010 & 8 & 0 & 8 \\
\hline
\end{tabular}

\section{Conclusion}

(1) Bit error rate and SNR Performance analysis of CDMA and IDMA is implemented using Matlab.

(2) Bit error rate and SNR Performance analysis of IDMA system over AWGN channel is done.

(3) Bit error Rate Performance analysis of IDMA system is better compared to CDMA. (BER for IDMA system is less than CDMA).

\section{Declarations}

\section{Source of Funding}

This research did not receive any specific grant from funding agencies in the public, commercial, or not-for-profit sectors.

\section{Competing Interests Statement}

The authors declare no competing financial, professional and personal interests.

\section{Consent for publication}

Authors declare that they consented for the publication of this research work.

\section{Availability of data and material}

Authors are willing to share data and material according to the relevant needs. 


\section{References}

1. Chirag R. Shah., A study on "Interleave division multiple access", Edelweiss publications, Volume 2, 2018.

2. Farheen Begum "Implementation of IDMA with multiple users in wireless communication system", Int. Journal of Computer Applications, Volume 134, 2016.

3. Dipesh Kamdar , "Simulation and performance analysis of IDMA in advance wireless communication system", Journal of Information Knowledge in Electronic and Communication, Volume 03, Issue 02, 2015.

4. Agarwal P., Pratap A., Shukla M., "BER analysis of power rotational interleaver on OFDM-IDMA system over powerline”, In: Hemanth J., Bestak R., Chen J.IZ. (eds) Intelligent Data Communication Technologies and Internet of Things. Lecture Notes on Data Engineering and Communications Technologies, Vol 57, Springer, 2021.

5. M.Moher and P.Guinand, "An iterative algorithm for asynchronous coded multi-user detection", IEEE Communication Letters., Vol.2, 229-331.

6. Kuldeep Choudhary, P.S.Sharma, "Interleave for IDMA technology: a comparison survey", Int J Advanced Res Computer Communication Eng., 1:2, 2012.

7. Rappport T.S., "Wireless communication: principles \& practice”, Prentice -Hall PTR, New Jersey.

8. Baier A, Fiebig U-C, Granzow W, Kotch W, Teder P and Thielecke J., "Design study for a CDMA-based third generation mobile radio system”, IEEE J. Select. Areas commun., Volume 12, Issue 4, 1994.

9. Morrow, R. K., “Accurate CDMA BER calculations with low computational complexity”, IEEE Transactions on Communications, 46(11), 1413-1417, 1998. 